\title{
Forensic Fellowship: Will a Clearer Process Produce More Forensic Fellows?
}

\author{
J. K. Hearn ${ }^{1}$ - A. B. McBride ${ }^{2}$. W. J. Newman ${ }^{1}$ \\ Received: 8 December 2019 / Accepted: 16 December 2019 / Published online: 23 December 2019 \\ (C) Academic Psychiatry 2019
}

\section{Commentary}

In recent years, general psychiatry residencies have experienced a surge in interest from increasingly qualified applicants [1]. During the same period, overall interest in psychiatric fellowships has remained stable or declined [2]. While it is possible that the increase in general psychiatry applications will later translate to an increase in fellowship applications, fellowship programs must consider multiple methods of garnering interest in subspecialty training as well as potential barriers to fellowship interest. Morris et al. [3] and Michaelsen et al. [4] outlined some challenges facing forensic psychiatry fellowships specifically. In reading these articles and others, the distinction between symptom and cause is not clearly apparent. For example, inconsistent referral streams could reflect limitations of a program's location (i.e., insufficient cases in the area) or may simply be the product of inconsistent availability of fellows to do the work. Such problems are likely idiosyncratic and unlikely to be resolved by any standardized approach across fellowships. Some of the challenges Morris et al. and Michaelsen et al. describe seem to have workable solutions, assuming training directors can agree upon the problems common to all fellowships. Both articles succeed in providing succinct, data-driven analysis of forensic psychiatry fellowships at both the applications and operations levels.

Michaelsen et al. stress the need for empirical studies to better understand the relationship between program-specific offerings and challenges common to forensic fellowships. Morris et al. echo this sentiment, considering the value of a FREIDA-like database in which detailed information about

W. J. Newman

will.newman@health.slu.edu

1 Saint Louis University School of Medicine, Saint Louis, MI, USA

2 University of California, Davis School of Medicine,

Sacramento, CA, USA applicants and fellowships could be collected and studied. We agree that without clear data, it is difficult to pinpoint and address the challenges facing forensic psychiatry training programs. Underscoring this point perfectly, the Michaelsen et al. and Morris et al. articles each cite a different number of forensic trainees (51 vs. 65) accepting positions for the same training period (2016-2017). If such basic information is not publicly available, we can only imagine the practical difficulties of conducting more systematic studies. Forensic training directors have repeatedly voted down initiating a match process. While one can debate the merits of a formal match into forensic psychiatry, it is difficult to find fault with calls for a more standardized and transparent application process. The Association of Directors of Forensic Psychiatry Fellowships (ADFPF) is actively in the process of developing a standard application for use by fellowship applicants. That is a first step toward creating a more clear, standardized process.

Many of the problems Michaelsen et al. outline are common to all psychiatric fellowships (e.g., sustaining funding, maintaining core training sites, and protected time for faculty). There are no simple solutions to these challenges. Cultivating interest of general psychiatry residents in the field of forensic psychiatry remains a critical goal. For any subspecialty to survive, it must continually demonstrate its value to prospective fellows. Residents may abandon pursuing a fellowship if they perceive that additional training is unnecessary, particularly if they have adequate experiences with psychiatric subspecialties during residency. This is primarily an issue of perception. We doubt that a prospective surgical resident would view general and vascular surgeons as interchangeable. It is less clear, however, how trainees view the psychiatric subspecialties relative to general psychiatry. All things considered, we believe that forensic psychiatry is well positioned to brand itself as a truly specialized branch of psychiatry.

Whether psychiatry trainees yet know this, they should all consider completing a forensic psychiatry fellowship. The overlap between psychiatry and the law is ubiquitous - from civil commitment, to malpractice standards, to evaluating 
dangerousness (and much more). Moreover, when a large percentage of psychiatry is now practiced in correctional or juvenile detention settings, it is critical that psychiatrists have sufficient forensic foundation to work with these underserved and high-need individuals. We must continue to promote that advanced training in forensic psychiatry through high-quality, ACGME-accredited forensic psychiatry fellowship training leads to knowledge and skills that are not in place upon graduating from a general psychiatry residency program.

How can we improve recruitment into this crucial subspecialty? First, start exposing psychiatry residents (and medical students) to forensic psychiatry early in their training. Forensic psychiatry faculty can find opportunities to teach students and residents, both incorporating forensic topics into the curriculum and modeling high-caliber educator and leadership skills. Second, make the link between general and forensic psychiatry. For example, as residents rotate through inpatient psychiatric facilities, teach them about the background of civil commitment laws in the state and country. When residents conduct a suicide risk assessment, emphasize the legal importance of adequate risk documentation and malpractice standards. For the child and adolescent psychiatry fellow evaluating a 10-year-old child at risk of entering the juvenile justice system, incorporate evidence-based treatments for minors with conduct disorder. Third, highlight the common goal of many individuals choosing careers in psychiatry-to help an often marginalized population with a high prevalence of serious mental illness by using ethical, effective, evidence-based treatments. When trainees realize that becoming an expert in mental health and the law aligns with their initial drive to practice psychiatry, we will train many more forensic psychiatrists. Forensic training leads to excellence in key areas that are generalizable to career success.

In just one additional year of training, forensic psychiatrists become far more comfortable embracing their identity as an expert witness. Some programs also encourage forensic fellows to present nationally or join national committees to advance goals they consider worthwhile. Additionally, forensic psychiatrists are trained to educate and effectively communicate complex psychiatric topics to individuals who may have little knowledge of mental health. By the time residents are trained in general psychiatry, they have undergone years of medical and mental health training. However, it takes a unique skillset to communicate the rich nuances of mental health where it intersects with the legal system to an audience without such extensive (or any) experience. Such a skillset can be applied in multiple settings from testifying in court to speaking with your local government representative about the merits of a new mental health bill to teaching a family about their child's psychological well-being. Further, forensic psychiatrists hone the skill of completing comprehensive evaluations using evidence-based practices and recommendations. They are taught to question and explore in detail each side of the story, producing objective opinions based on extensive data. This practice may be more difficult to accomplish in clinical psychiatry given limitations in time and available resources. Ultimately, we believe that healthy forensic fellowships add benefits to associated general psychiatry residency programs and to the field of psychiatry as a whole. For forensic psychiatry fellowships to thrive, problems common to all programs must be identified in a data-driven fashion. Morris et al. and Michaelsen et al. succeed in outlining these common challenges.

\section{Compliance with Ethical Standards}

Disclosure On behalf of all authors, the corresponding author states that there is no conflict of interest.

\section{References}

1. Agapoff JR, Tonai C, Eckert DM, Gavero G, Goebert DA. Challenges and perspectives to the rise in general psychiatry residency applications. Acad Psychiatry. 2018;42:674-5.

2. Agapoff JR, Olson DK. Challenges and perspectives to the fall in psychiatry fellowship applications. Acad Psychiatry. 2019;43:425-8.

3. Morris NP, Hirschtritt ME, Edwards ML. Variations in application instructions for forensic psychiatry fellowships. Acad Psychiatry. 2019.

4. Michaelsen K, Piel J, Kopelovich S, Reynolds S, Cowley D. A review of forensic fellowship training: similar challenges, diverse approaches. Acad Psychiatry. 2019.

Publisher's Note Springer Nature remains neutral with regard to jurisdictional claims in published maps and institutional affiliations. 